\title{
A Method of Equalizing Frequency Responses of Adaptive Antenna Array Channels
}

\author{
Alexey A. Erokhin, Evgeniy R. Gafarov, Pavel V. Shtro \\ Institute of Engineering Physics and Radio Electronics \\ Siberian Federal University \\ Krasnoyarsk, Russia \\ AErokhin@sfu-kras.ru
}

\begin{abstract}
A method for equalizing frequency responses of the adaptive antenna array channels is shown. This method does not require a reference signal or some additional antennas. Equalizing is performed on the received signal (like interference signal).
\end{abstract}

Keywords-antenna arrays; antenna beam patterns; adaptive array; equelizer

\section{INTRODUCTION}

For interference suppression we need create a minimum of the beam pattern in it direction. To create a deep minimum necessary to satisfy the following conditions:

1. The dynamic range of the receiving equipment sufficient for reception of the desired signal and interference without distortion.

2. Number of interference should not exceed $(N-1)$, where $N$ - number of elements antenna array.

3. Amplitude-frequency and phase-frequency responses of channels must be identical.

Implementation of the first and second conditions is quite achievable. Implementation of the third condition is a serious problem, because amplitude-frequency and phase-frequency responses of channels are varying. Also it is depend on the interference position in space and frequency responses of channels may be varied from time $[1,2]$. Thus, we need the device that can equalize channels frequency responses.

\section{ADAPTIVE FILTER}

Let us consider an adaptive filter that shown in Fig. 1. Weights coefficients of the adaptive filter will be set up according with signal of the first channel $\left(\dot{x}_{1}(t)\right)$.

The adaptive filter (equalizer) input signal is a second channel signal of the antenna array $\left(\dot{x}_{2}(t)\right)$. Then an error signal has a form:

$$
\dot{e}(t)=\dot{x}_{1}(t)-\dot{y}(t)=\dot{x}_{1}(t)-\sum_{m=1}^{M} \dot{h}_{m} \dot{x}_{2}(t-m),
$$

where $M-$ number of filter coefficients, $\dot{h}_{m}$ - adaptive filter weight coefficient, $t$ - discrete time; dot above symbols

The reported study was funded by RFBR and Government of Krasnoyarsk Territory according to the research project № $16-47-243016$ denote complex value.

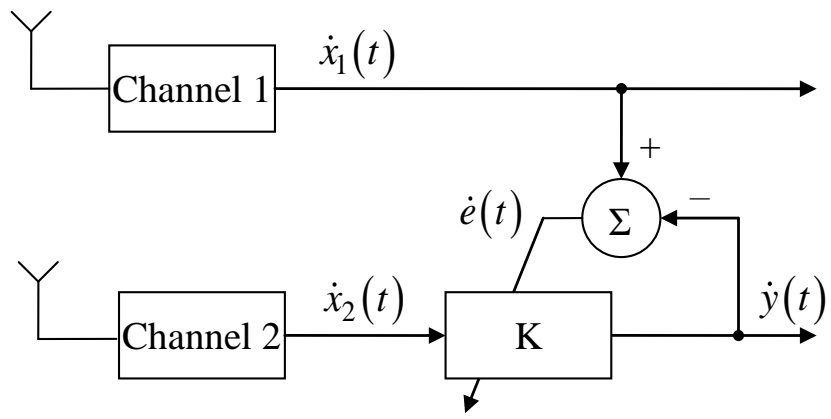

Fig. 1. Antenna array with adaptive filter.

It is known that optimal weight coefficients in minimum mean-square sense are solution of the Wiener-Hopf equation [3]:

$$
\dot{\mathbf{h}}_{\text {opt }}=\dot{\mathbf{R}}^{-1} \dot{\mathbf{r}},
$$

where $\quad \dot{\mathbf{R}}=E\left\{\dot{x}_{2}(t) \dot{x}_{2}^{H}(t)\right\} \quad-\quad$ covariance matrix, $\dot{\mathbf{r}}=E\left\{\dot{x}_{1}(t) \dot{x}_{2}(t)\right\}$ - cross-correlation vector; bold symbols denote vectors and matrices.

\section{EQUALIZING METHOD}

A frequency responses equalizing by input signal of the antenna array, i.e. by the interference signal; we consider that useful signal power lower than interference signal power or its absence while adaptive filter sets coefficients. A signal receiving by first channel of the antenna array is the reference signal for adaptive filter (equalizer); in common case is an interference signal that is a random signal with unknown direction of arrival. Then optimal weight coefficients for array channels (except first channel, because this is reference channel) calculated by adaptive algorithm, in this case is a Wiener-Hopf solution.

Signals received by the antenna array has a different phase shifts. It is caused by the geometry of the array and direction of arrival of the signals. These phase shifts will be reset by the equalizer (set in zero value) in channels of the antenna array, also in normal direction to the plane of array will set a "zero" of the beam pattern. This effect is shown in Fig. 2, as an 
example we use the uniform linear array that has 4 elements and distance between adjacent elements is $\lambda / 2$. Direction of arrival of the interference signal is $40^{\circ}$.

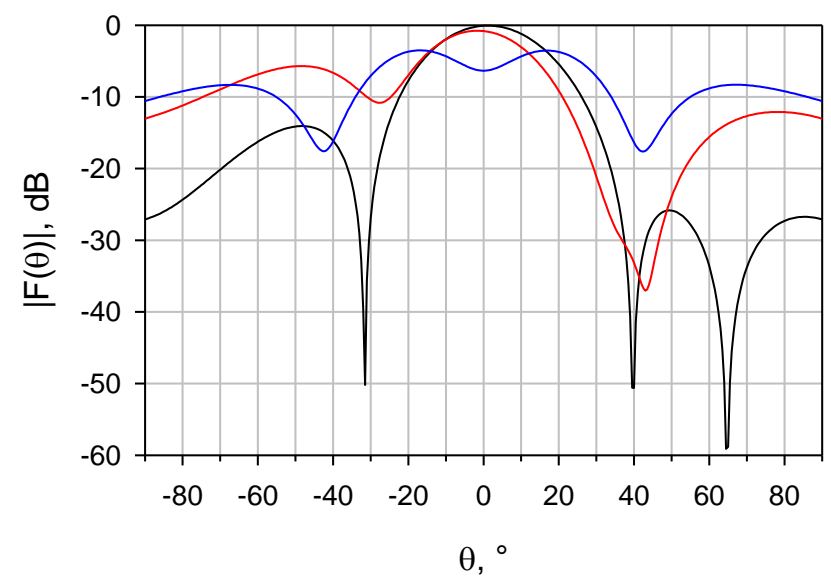

Fig. 2. Beam pattern with a "zero" in normal direction to the plane of array.

To avoid this effect it is necessary to introduce in the output signal of the adaptive filter phase delays corresponding to the arrival direction of the interference signal. According to the principal component analysis [4] eigenvectors of the covariance matrix will determine the phase shifts corresponding to the direction of arrival of the interference signal. In this case, the beam pattern is no "zero" in normal direction to the plane of the array (see Fig. 3).

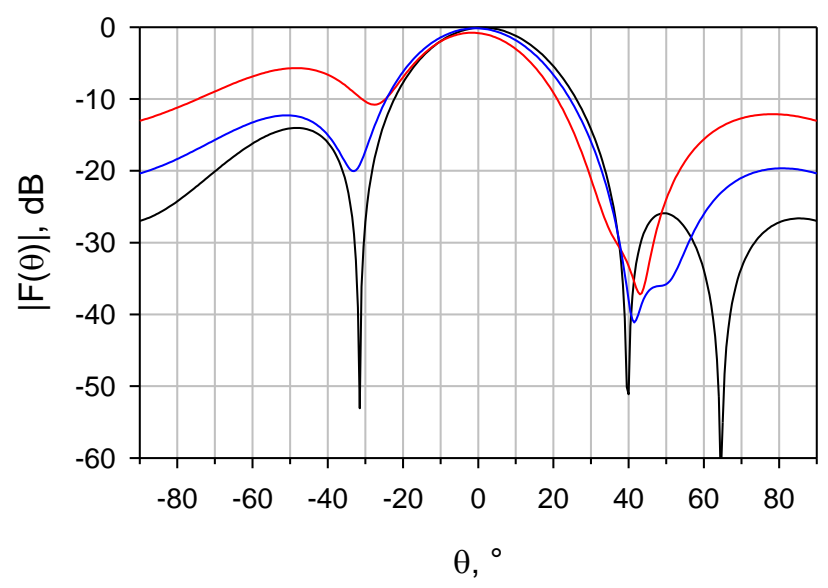

Fig. 3. Beam pattern with phase shifts compensation.

Legend for Fig. 2 and Fig. 3:

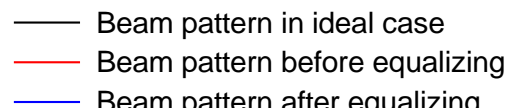

On the basis of the above we can change scheme of the antenna array with adaptive filter (Fig. 1). These changes are shown in Fig. 4.

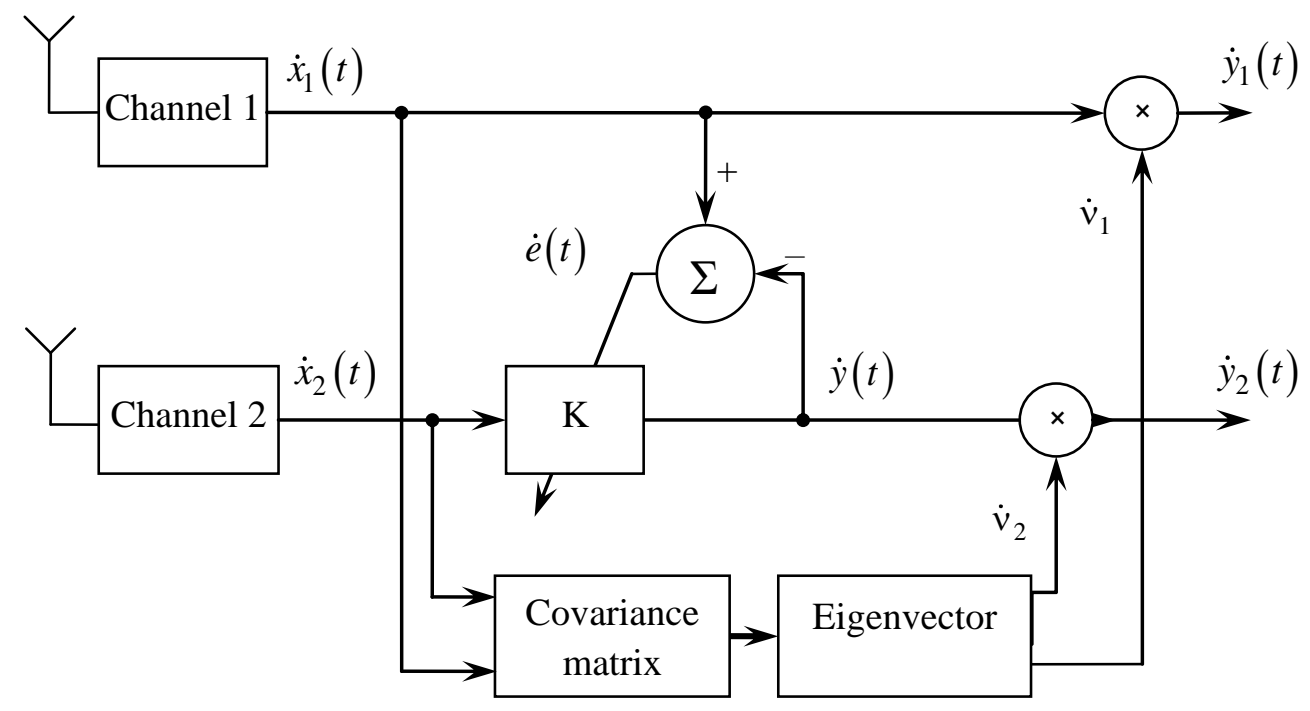

Fig. 4. Antenna array with phase shifts compensation.

According to the scheme shown in Fig. 4, at the beginning, we find the first eigenvector $\dot{\mathbf{v}}$ by the input signal $\left(\dot{x}_{n}(t)\right)$. Elements $\dot{v}_{m}$ of the eigenvector are coefficients for the phase shift compensation. We can accumulate eigenvector by some number of input samples and then not calculate it. Next, multiply equalized signals (the adaptive filter output signal $\dot{y}(t))$ to the corresponding element of the eigenvector. First channel signal necessary multiply to first eigenvector element too. By signals $\dot{y}_{n}(t)$ we can calculate covariance matrix and vector of optimal weights for interference suppression.

\section{NUMERIC SIMULATIONS}

Let us define suppression coefficient as ratio of signal power received by one antenna to signal power at the array output in interference suppression mode [5]. It is interesting the number of adaptive filter weight coefficients required for complete suppression of interference. Consider the 4-element 
uniform linear antenna array. Distance between adjacent elements is $\lambda / 2$. In this simulation we assume that power of the useful signal is very small, i.e. signal to noise ratio is $\mathrm{SNR}=$ $90 \mathrm{~dB}$. And interference to noise ratio is $\mathrm{INR}=40 \mathrm{~dB}$. So we can draw a dependence of the suppression coefficient from number of the equalizer weight coefficients. This dependence is shown in Fig. 5. The results are averaged over the 1000 simulations.

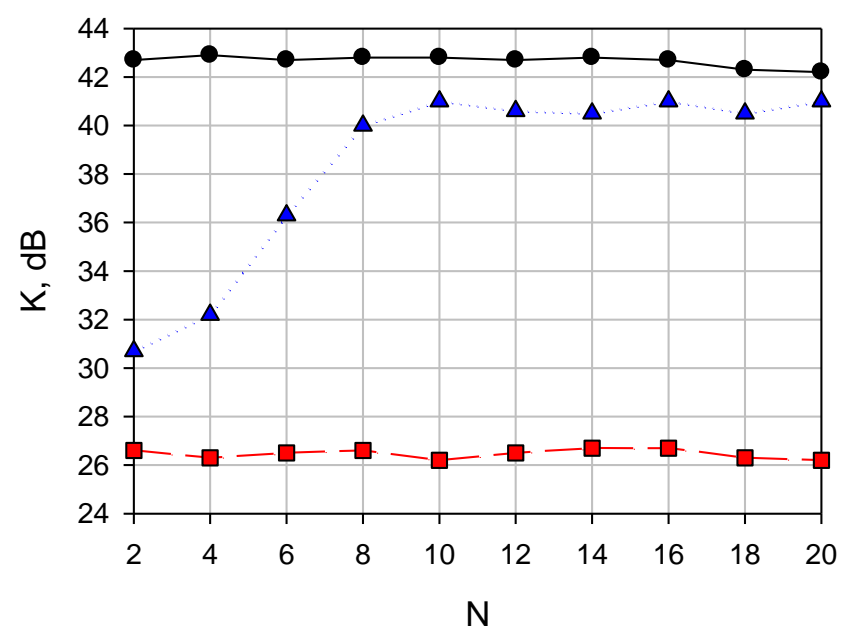

Fig. 5. Suppression coefficient of the adaptive antenna array with phase shifts compensation.

Legend for Fig. 5:

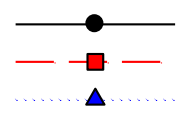

Suppression coefficient in ideal case Suppression coefficient before equalizing Suppression coefficient after equalizing

As we can see from Fig. 5 is enough to use 10 weight coefficients for interference suppression.

In Fig. 6 and Fig. 7 shows spectra of signals of the adaptive antenna array. In this simulation we assume that number of weight coefficients is 12 and $\mathrm{SNR}=-30 \mathrm{~dB}$, $\mathrm{INR}=20 \mathrm{~dB}$.

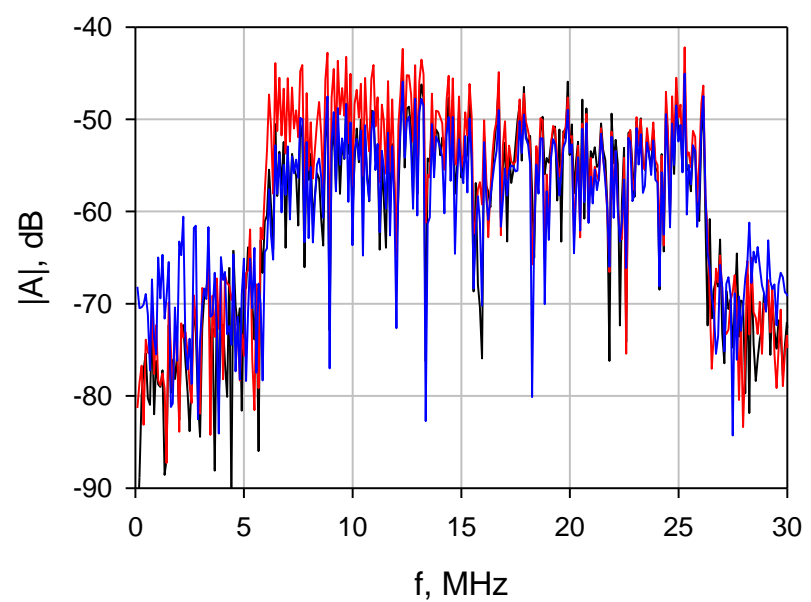

Fig. 6. Amplitude spectra of input signals

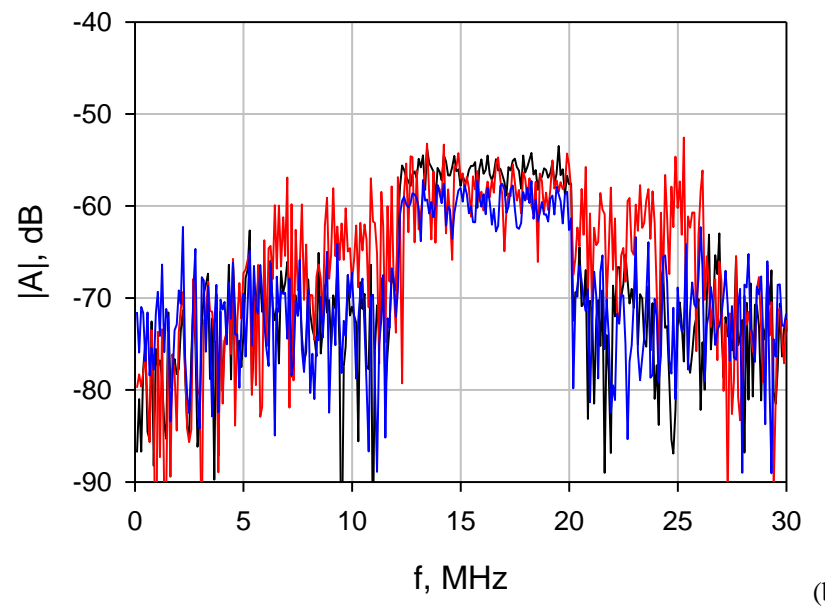

(b)

Fig. 7. Amplitude spectra of output signals.

Legend for Fig. 6 and Fig. 7:

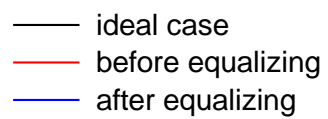

As we can see from Fig. 6 and Fig. 7 amplitude spectra for input (interference) and output (useful) signals are equalized.

\section{SUMMARY}

The channel equalization method can be applied to improve the characteristics of interference suppression in adaptive antenna array and for equalizing frequency response of non adaptive arrays.

\section{REFERENCES}

[1] Nishimori K.,Cho K.Takatori Y., Hori T. "A new calibration method of adaptive array for TDD systems", IEEE Antennas and Propagation Society International Symposium, Jul 11-16 1999. Vol. 2. pp. 14441447.

[2] Nishimori K.,Cho K.,Takatori Y.,Hori T., "Automatic calibration method of adaptive array for FDD systems", IEEE Antennas and Propagation Society International Symposium, Jul 16-21 2000. Vol. 2. pp. 910-913.

[3] Farhang-Boroujeny, B., Adaptive filters: theory and applications. Second edition. John Wiley \& Sons Ltd, 2013. 802 p.

[4] Gorban A.N., Kégl B., Wunsch D.C., Zinovyev A., Principal Manifolds for Data Visualization and Dimension Reduction, Series: Lecture Notes in Computational Science and Engineering. Springer, 2008. 360 p.

[5] Erokhin A.A., Salomatov Yu.P., "Statistical Analysis of the Adaptive Antenna Array Power Pattern", 2015 International Siberian Conference on Control and Communications (SIBCON). Proceedings., May 2015. 